\title{
Discrimination of Cycling Patterns Using Accelerometric Data and Deep Learning Techniques
}

\author{
Aleš Procházka • Hana Charvátová • Oldřich Vyšata • \\ Delaram Jarchi · Saeid Sanei
}

Received: date / Accepted: date

\begin{abstract}
The monitoring of physical activities and recognition of motion disorders belong to important diagnostical tools in neurology and rehabilitation. The goal of the present paper is in the contribution to this topic by (i) analysis of accelerometric signals recorded by wearable sensors located at specific body positions and by (ii) implementation of deep learning methods to classify signal features. This paper uses the general methodology to analysis of accelerometric signals acquired during cycling at different routes followed by the global positioning system (GPS). The experimental dataset includes 850 observations that were recorded by a mobile device in the spine area (L3 verterbra) for cycling routes with the different slope. The proposed methodology includes the use of deep learning convolutional neural networks with five layers applied to signal values transformed into the frequency domain without
\end{abstract}

A. Procházka ( $)$

University of Chemistry and Technology in Prague, Department of Computing and Control Engineering, 16628 Prague 6, \& Czech Technical University in Prague, Czech Institute of Informatics, Robotics and Cybernetics, 16636 Prague 6, Czech Republic, E-mail: Ales.Prochazka@vscht.cz

H. Charvátová

Tomas Bata University in Zlin, Faculty of Applied Informatics, 76001 Zlin, Czech Republic, E-mail: charvatova@utb.cz

O. Vyšata

Charles University in Prague, Department of Neurology, Faculty of Medicine in Hradec Králové, 50005 Hradec Králové, Czech Republic, E-mail: oldrich.vysata@fnhk.cz

\section{Jarchi}

University of Essex, School of Computer Science and Electronic Engineering, Colchester CO4 3SQ, United Kingdom, E-mail: delaram.jarchi@essex.ac.uk

S. Sanei

Nottingham Trent University, School of Science and Technology, United Kingdom, E-mail: saeid.sanei@ntu.ac.uk specification of any signal features. The accuracy of discrimination between different motion patterns for the uphill and downhill cycling and recognition of 4 classes associated with different route slopes was $96.6 \%$ with the loss criterion of 0.275 for sigmoidal activation functions. These results were compared with those evaluated for selected sets of features estimated for each observation and classified by the support vector machine, Bayesian methods, and the two-layer neural network. The best cross-validation error of 0.361 was achieved for the two-layer neural network model with the sigmoidal and softmax transfer functions. Our methodology suggests that deep learning neural networks are efficient in the assessment of motion activities for automated data processing and have a wide range of applications, including rehabilitation, early diagnosis of neurological problems, and possible use in engineering as well.

Keywords multimodal signal analysis · computational intelligence $\cdot$ machine learning · deep neural networks · accelerometers $\cdot$ classification $\cdot$ motion monitoring

\section{Introduction}

Computer assisted monitoring of motion activities [57, $34,33,36,31]$ allows us to improve the quality of life in many different areas, including health care and personal fitness. The need for early detection of different pathological situations motivates the study of specific methods to examine motion disorders in clinical neurological practice $[13,2]$ using wearable sensors $[38,58,54]$ and vision based techniques. However, the use of video camera systems is limited to the direct visibility of the object [22], while wearable devices can directly translate human motion into signal patterns for the following mathematical processing and activity recognition. Re- 

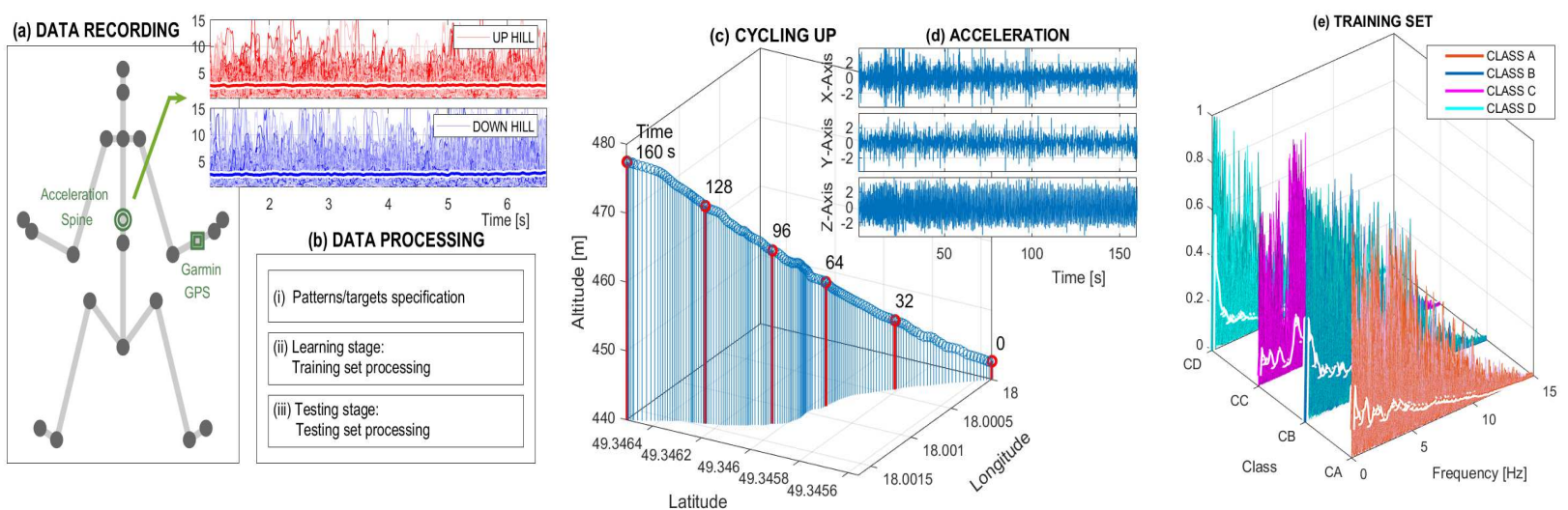

Fig. 1 Principle of data processing presenting: (a) accelerometric data acquisition using a wearable sensor in the spine area of the body with modules of these data for the selected set of experiments for uphill and downhill cycling, (b) data processing steps, (c) a sample route $190 \mathrm{~m}$ long recorded by the GPS system, (d) associated accelerometric data acquired by 3-axis sensor inside the smartphone, and (e) spectral values related to accelerometric data recorded during the uphill (Class CA), downhill $(\mathrm{CB})$, steep uphill $(\mathrm{CC})$, steep downhill $(\mathrm{CD})$ cycling with their mean values.

cent advances in sensor technology and wireless communication systems allow us to use smartphones, smartwatches, and global positioning systems (GPS) for human motion monitoring (Fig. 1), assessment of sport activities $[46,9,44]$, detection of movement symmetry [47], and gait analysis [26]. Associated methods of computational intelligence and deep learning allow us to evaluate these data with satisfactory accuracy in many applications $[53,1,35]$.

Classical clustering, segmentation, and classification methods, including decision tree (DT), $k$-nearest neighbour (k-NN), support vector machines (SVM), Bayesian methods and the two-layer neural network (NN) algorithms with different transfer functions, are often used in this area $[45,4,24]$. Another more complex approach is based on the use of deep learning methods that are applied to optimise the multilayer NN for the construction and evaluation of motion models. The problem of limited dataset size in these cases [60] are sometimes solved by augmentation methodology, which enables us to enlarge the size of training datasets, to reduce the class imbalance, and to minimise scalability issues [37]. This approach has been tested in many areas, including gait recognition algorithms $[8,26]$ and in dentistry for teeth category classification using convolutional NNs.

The classification of signal segments of biomedical signals is performed by classical clustering methods, by deep learning algorithms [51,42,16], and by specific discrimination methods [30]. The time, frequency, and scale domains are often used in these cases as the initial information sources for human activity monitoring. Brain functionality is analysed in some studies [12] for sleep scoring or emotion recognition. In addition, ECG signals are often analysed in a similar way.

Deep learning methods $[19,50,55,6]$ are often used as general mathematical tools in many areas, including engineering, biology [3], biomedicine, and neurology. On the other hand, alternative specific methods of signal and image processing, modelling [61,25,29,17], detection of multidimensional signal components, and signal segments recognition are often related to specific problems. Applications of both approaches include analysis of thermal processes and energy resources $[15,56]$, biometrics [28], stomatology [21], human activity recognition $[18,59]$, or interpretation of natural kinematics for human authentication [40]. While deep learning methods are able to process a complete signal segment or an image as a general body, specific methods are based on a selection of appropriate feature in time, or selected functional domains in many cases.

The present paper is devoted to the application of deep neural networks (DNNs) for motion analysis [20] closely related to rehabilitation and evaluation of motion disorders. The goal of the study is in the contribution to these topics and analysis of accelerometric signals recorded by wearable sensors located at specific body positions. The analyzed signals include those acquired by three-axis accelerometers during the uphill and downhill cycling on routes with different slopes (Fig. 1(c)). All datasets were acquired by a smartphone sensor that was located on the spine. This position was selected as the best location [10] enabling to distinguish different cycling activities with the highest accuracy.

The proposed methodology forms a contribution to the analysis of human motion data acquired during different movement events including sport activities and neurological disorders [13]. Accelerometric data analyzed in the present paper form a simple and cheap information source while in other applications different sensors can be used. Their selection includes video and depth cameras for gait analysis [49], GPS systems for cycling monitoring [9], thermal cameras [44] for breath- 
ing evaluation, heart rate [10] and further biosensors for motion analysis during the sleep.

Results of the study contribute to the analysis of accelerometric data for motion monitoring and they include the comparison of the deep learning (DL) with further classification methods for recognition of different motion patterns during cycling associated with selected route slopes. From the more general point of view, the paper contributes to classification of vibrations associated with diagnosis of motion disorders in neurology [13] or during sport activities, and for analysis of engineering systems as well.

\section{Methods}

\subsection{Data Acquisition}

Measuring accelerometric data during different cycling activities allows the motion patterns that are important for fitness evaluation to be recognised and for movement disorders to be monitored. All signals were recorded by wearable sensors inside a smartphone that was located in the spine area (L3 lumbar vertebra) of the body, as shown in Fig. 1(a). This selection resulted from the previous research $[13,10,52,38]$ that compared classification results for sensors located at different parts of the body simultaneously recorded by 31 sensors of the perception neuron [5].

The three-axes accelerometric data and their time stamps were recorded during uphill and downhill cycling at routes of the different slopes to monitor physical activity [2]. Fig. 1(c) presents one of cycling routes with associated time stamps. The terrain slope was evaluated from the global positioning system (GPS) with data recorded by the GARMIN smartwatch system.

Our data set included 850 segments that resulted from 170 cycling experiments with each of them divided into 5 parts of the same length. The average sampling frequency of accelerometric data acquisition was $115 \mathrm{~Hz}$. Table 1 presents details of these sets including time lengths of experiments for the uphill, downhill, steep uphill, and steep downhill cycling.

The whole set of experiments was reduced by $3.53 \%$ of unreliable measurements to 820 records, which were

Table 1 Statistics of experiments including the average route time lengths for selected terrain slopes with their standard deviations (STD).

\begin{tabular}{|c|c|c|}
\hline \multirow{2}{*}{ Class } & \multicolumn{2}{|c|}{ Route Time Length [s] } \\
\hline & Mean & $S T D$ \\
\hline CA - uphill & 157.1 & 2.9 \\
\hline CB - downhill & 59.5 & 1.5 \\
\hline CC - steep uphill & 165.7 & 7.6 \\
\hline CD - steep downhill & 77.7 & 6.5 \\
\hline
\end{tabular}

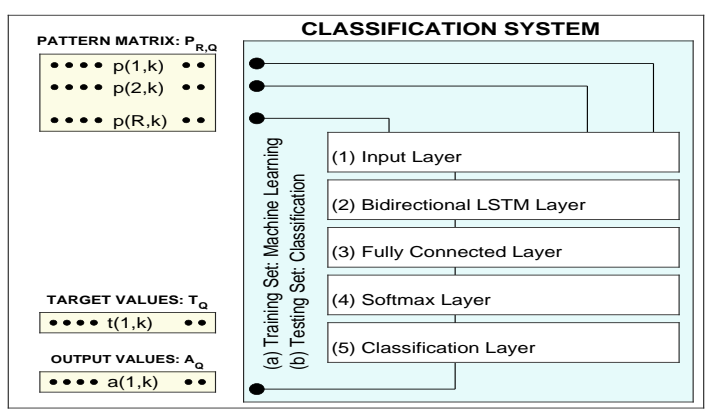

Fig. 2 Principle of data processing and the classification system based on the DNN with five layers.

in the range of the selected multiple of standard deviation from the mean value for each class. Each experiment that belonged to specific class was associated with the slope value evaluated from GPS data. Table 2 presents the summary of signal segments that we used.

All of the cycling experiments were divided into the training and testing sets with $90 \%$ and $10 \%$ of observations, respectively. The selection of signal segments was done randomly but with the balanced number of segments belonging to classes $\mathrm{CA}, \mathrm{CB}, \mathrm{CC}$ and $\mathrm{CD}$. The comparison of classification accuracies was then evaluated both for the training and testing sets.

Fig. 1(e) presents spectral values (evaluated from time domain values presented in Fig. 1(d) of training sets of observations belonging to classes $\mathrm{CA}, \mathrm{CB}, \mathrm{CC}$ and $\mathrm{CD}$, with their mean values that represent typical curves of spectral components individual classes. For each experiment of the specific class, associated features were estimated. The whole set of experiments was then analysed using statistical tools and selected classification methods were applied to the testing set.

Table 2 Statistics of accelerometric data including recorded (Rec.) and extracted (Extr.) number of segments for different terrain slopes with their standard deviation (STD), as recorded by the Garmin GPS system.

\begin{tabular}{lccccc}
\hline \multirow{2}{*}{ Class } & \multicolumn{2}{c}{ Segments } & & \multicolumn{2}{c}{ Slope } \\
\cline { 2 - 3 } \cline { 6 - 7 } & Rec. & Extr. & & S[\%] & STD \\
\hline CA - uphill & 205 & 199 & & 10.3 & 3.3 \\
CB - downhill & 210 & 205 & & -9.4 & 3.9 \\
CC - steep uphill & 210 & 201 & & 19.8 & 3.4 \\
CD - steep downhill & 225 & 215 & & -18.7 & 3.4 \\
\hline
\end{tabular}

\subsection{Feature Extraction and Classification}

The data processing presented in Fig. 1(b) included statistical analysis of individual accelerometric records for each class (Class CA: uphill cycling, Class CB: downhill cycling, Class CC: steep uphill cycling, Class CD: steep downhill cycling) recorded with a sampling frequency $f_{s}$, depending upon the used smartphone technology, and varying between 80 and $150 \mathrm{~Hz}$ in many cases. The 
standard deviation of differences between each measurement and their mean values was then used as a measure to extract records affected by gross measurement errors.

The initial data processing included data interpolation, filtering [31], and resampling to eliminate slightly changing sampling period during data acquisition. This process also included digital filtering to reject slowly changing mean data values and high frequency components. In this stage, the passband finite impulse response (FIR) filtering of an order $M$ was applied to each signal segment $\{r(n)\}_{n=0}^{N-1}$ for selected cutoff frequencies to evaluate a new sequence $\{s(n)\}_{n=0}^{N-1}$.

In the next step, the modulus $x(n)$ of the accelerometric data was evaluated from the components $s_{x}(n)$, $s_{y}(n)$, and $s_{z}(n)$ recorded in three directions using the following relation

$x(n)=\sqrt{s_{x}(n)^{2}+s_{y}(n)^{2}+s_{z}(n)^{2}}$

for all values $n=0,1,2, \cdots, N-1$ in each segment $N$ values long. Each record was then transformed by the discrete Fourier transform (DFT) into the frequency domain, which was used to separate the signals into individual classes.

The estimation of signal features can be performed both in the time and transform domains using either the discrete Fourier, wavelet $[27,11]$ or Radon transforms. In these domains, the time dependent features can be evaluated and spectrograms or scalograms can be used to optimise the structure and coefficients of complex mathematical models for data classification. Our proposed approach used the simple discrete Fourier transform of each time segment $\{x(n)\}_{n=0}^{N-1} N$ samples long forming the sequence $\{X(k)\}_{k=0}^{N-1}$ for $k=0,1, \cdots, N-1$ and defining frequency values $f_{k}=k / N f_{s}$, which appeared to be sufficient in the given application.

The pattern matrix used for the following classification was defined for the training set using randomly chosen $90 \%$ measured values by two methods:

- Deep learning: each column of the pattern matrix included all of the values of each spectral curve in the training set with a frequency resolution of $f_{s} / K$ $\mathrm{Hz}$ for the chosen total number of $K$ frequency samples (selected as $K=1024$ ).

- Standard classification methods: each column of the pattern matrix included specific features that were associated with each sample in the training set, which were estimated in the frequency domains.

Target values associated with each column of the pattern matrix were in both cases specified by the positive slope (Classes CA, CC) and the negative slope (Classes $\mathrm{CB}, \mathrm{CD})$ value.

Accelerometric data segments in the time-domain had different lengths but the modified DFT provided the same selected number $K=1024$ of frequency samples in the range of $\left\langle 0, f_{s}\right\rangle \mathrm{Hz}$ for the selected sampling frequency $f_{s}=60 \mathrm{~Hz}$ (after resampling) which allowed the use of the constant number of input elements $R$ of the deep learning network presented in Fig. 2(b). The selection of $R=K / 4=256$ enabled the use of the constant number of input network coefficients that covered frequency components $\langle 0,15\rangle \mathrm{Hz}$.

The features based upon spectral components in the frequency domain included:

(i) The relative mean power in the range $\left\langle f_{a 1}, f_{a 2}\right\rangle$,

(ii) The relative mean power in the range $\left\langle f_{a 3}, f_{a 4}\right\rangle$.

Each of spectral features of a signal segment $\{x(n)\}_{n=0}^{N-1}$ $N$ samples long was evaluated using the discrete Fourier transform in terms of the relative power $P$ in a normalised frequency band $\left\langle f_{a i}, f_{a j}\right\rangle$, as follows:

$P=\frac{\sum_{k \in \Phi}|X(k)|^{2}}{\sum_{k=0}^{N / 2}|X(k)|^{2}}$

where $\Phi$ is the set of indices for frequencies $f_{k} \in\left\langle f_{a i}, f_{a j}\right\rangle$.

Figure 3 presents the distribution of selected couples of features used for the following classification using the relative mean power in the frequency range $\langle 3,8\rangle \mathrm{Hz}$ versus the relative mean power in the frequency range $\langle 8,15\rangle \mathrm{Hz}$ with centers and $c$-multiples of standard deviations of each class for $c=0.2, c=0.5$ and $c=1$. Feature clusters for steep hill data processing are more compact and better separated owing to their physiological background.

Data segments affected by gross errors were eliminated from further processing. As a criterion of this procedure the Euclidean distances of spatial positions of features from the centers of gravity, their mean and standard deviations were used.
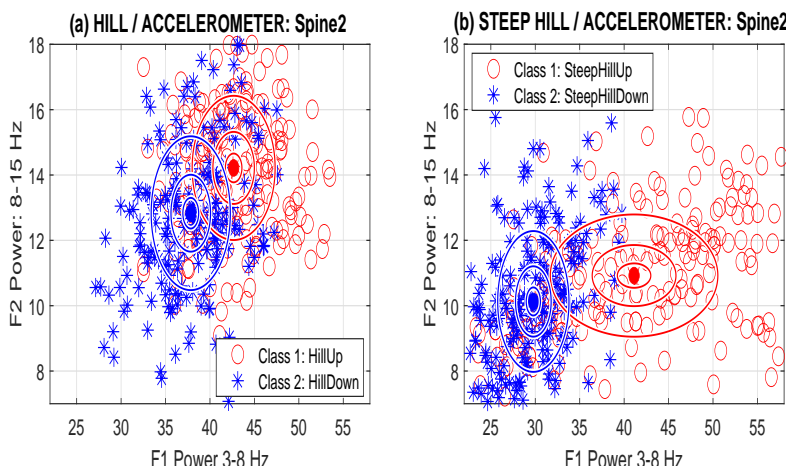

Fig. 3 The distribution of features using the relative mean power in the frequency range $\langle 3,8\rangle \mathrm{Hz}$ versus the relative mean power in the frequency range $\langle 8,15\rangle \mathrm{Hz}$ with centers and $c$-multiples of standard deviations of each class for $c=$ $0.2, c=0.5$, and $c=1$ for (a) uphill/downhill cycling and (b) steep uphill/downhill cycling using a selected accelerometer position. 


\subsection{Movement Recognition}

Selected $Q$ feature vectors associated with signal segments were used as column vectors of the pattern matrix $\mathbf{P}_{R, Q}$. The target matrix $\mathbf{T}_{S, Q}$ of desired class probabilities was then applied $[19,23]$ to classify all of the $Q$ feature vectors into $S$ classes. The results of the deep learning system were compared with those evaluated by classical systems, which included a SVM, a Bayesian method, and a two-layer NN.

The matrix $\mathbf{A}_{S, Q}$ of output values of the selected classification system are evaluated through the system description function $f$ by the following relation:

$\mathbf{A}_{S, Q}=f\left(\mathbf{P}_{R, Q}\right)$

During the learning stage, system coefficients are optimized to obtain its output values $\mathbf{A}_{S, Q}$ as close as possible to target values $\mathbf{T}_{S, Q}$ for each column vector of the pattern matrix $\mathbf{P}_{R, Q}$. The values of the output layer with the softmax function, based on the Bayes' theorem [48], provide the probabilities of each class.

The DNN system [39] uses $R$ frequency components of signal segments in each pattern vector. The classification system included the input layer, bidirectional long short term memory (LSTM) [43], fully connected layer, softmax layer and the classification layer [19,41], as presented in Fig. 2(b). The deep learning strategy was used to minimize the selected criterion function.

The performance of classification models is often evaluated by the log-loss function, which takes into account the probability that is assigned to the estimation of the target value. This can be evaluated by the following relation

$L L=-\frac{1}{Q} \sum_{i=1}^{Q}(t(i) \log (p(i))+(1-t(i)) \log (1-p(i)))$

where $t(i)$ stands for the binary output to be predicted, $p(i)$ stands for the probability assigned by the model, and $Q$ is the number of target values. Coefficients of the classification system are then optimised during the machine learning process to minimise the value of this criterion. Its strength lies in the fact that the log-loss function combines the correct and strong prediction. In addition, as a measure of predictive inaccuracy, it should be as low as possible.

The standard two-layer NN system is formed by a simplified system defined by Eq. 3 with $R=2$ features in this case. The network coefficients include values of the first and the second layer with the sigmoidal transfer function in the first layer. These coefficients are optimized to obtain probabilities $\mathbf{A}_{S, Q}$ close to target values $\mathbf{T}_{S, Q}$ using the softmax function as in the DNN case. Both the accuracies and the cross-validation errors were used to evaluate the individual results.
Table 3 The confusion matrix for the evaluation of a model classifying pattern vectors belonging to true (target) classes and estimating their (predicted) classes for the multi-class classification into $S$ categories.

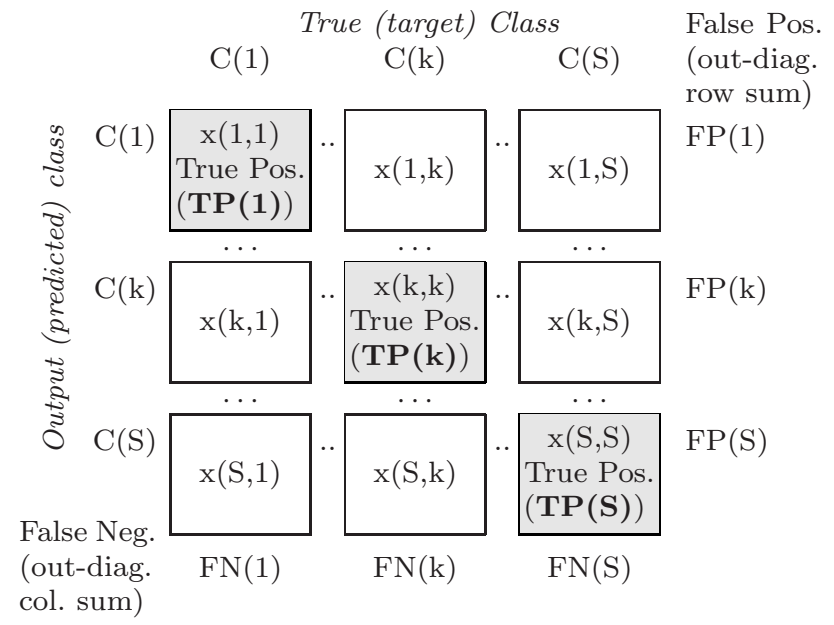

The selection of the classification model is closely related to the application area and the number of pattern values used for system optimisation in the learning stage. In many applications, simple classification systems provide sufficient results. However, in the case of more complicated patterns, DNNs with specific (convolutional) layers [19] are often used for effective decision making with the sufficient generalisation ability.

The evaluation of the classification results was performed by the multi-class Receiver Operating Characteristic (ROC) $[14,7,45]$ to illustrate the performance of the classifier system, and by a confusion matrix presented in Table 3 for classification into $\mathrm{S}$ classes. The ROC analysis was performed on the basis of a pairwise comparison of one class against all other classes [32].

The associated common performance metrics used in the multiclass confusion matrix include the following ones:

- Precision of class $k$ (pos. predict. value) - Probability of correct classification of class $k$ related to the number of instants classified to class $k$

$P P V(k)=\frac{T P(k)}{T P(k)+F P(k)}$

- Sensitivity of class $k$ (True positive rate, recall) Probability of correct classification of class $k$ related to the number of instants belonging to class $k$

$T P R(k)=\frac{T P(k)}{T P(k)+F N(k)}$

- Specificity of class $k$ (True negative rate) - Probability of incorrect classification of class $k$ related to the number of instants not classified to class $k$

$T N R(k)=\frac{T N K(k)}{T N K(k)+F P K(k)}$,

where $T N K(k)=\sum T P(j), F P K(k)=\sum F P(j), j \neq k$ 


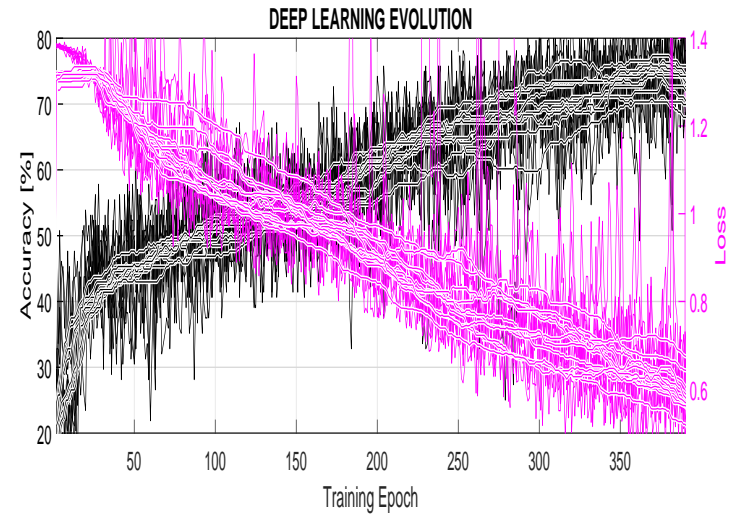

Fig. 4 Results of the initial 400 epochs of the deep learning optimisation presenting the training accuracy and the loss for 10 experiments with randomly selected initialisations.

- False positive rate - Probability of positive classification for the negative set (1-specificity)

$$
F P R(k)=\frac{F P K(k)}{T N K(k)+F P K(k)}
$$

- Accuracy - Probability of global correct classification

$$
A C C=\frac{\sum_{k} T P(k)}{\sum_{k}(T P(k)+F P(k))}
$$

\section{Results}

The accelerometric data were classified into four classes (class CA: uphill cycling, class CB: downhill cycling, class CC: steep uphill cycling, class CD: steep downhill cycling) using several methods. Figure 4 shows the results of the initial 400 epochs of the deep learning optimisation process, presenting the training accuracy and the loss for different randomly selected initialisations of the whole model during 10 experiments. The goal of this process was to optimise the network to maximise the accuracy and to minimise the loss during individual epochs. This optimisation was performed using the mathematical and software environment of the Matlab2020a system.

Table 4 presents the accuracy $A C[\%]$ and the loss value $L V$ for 10 deep learning experiments for different network initialisations presenting the final value $(\mathrm{F})$ after 400 learning epochs and its mean (F30) evaluated from 30 last epochs. Training was performed for the training set of values presented in Fig. 2(a) and verification for the different set of $10 \%$ of experiments randomly chosen from all observations. The mean value of the accuracy and the loss evaluated from last 30 epochs was $74.6 \%$ and 0.63 , respectively, with its standard deviation reduced by two orders with respect to the evaluation of final values only.

The optimization process of the DNN structure presented in Fig. 2 for the chosen initial learning rate

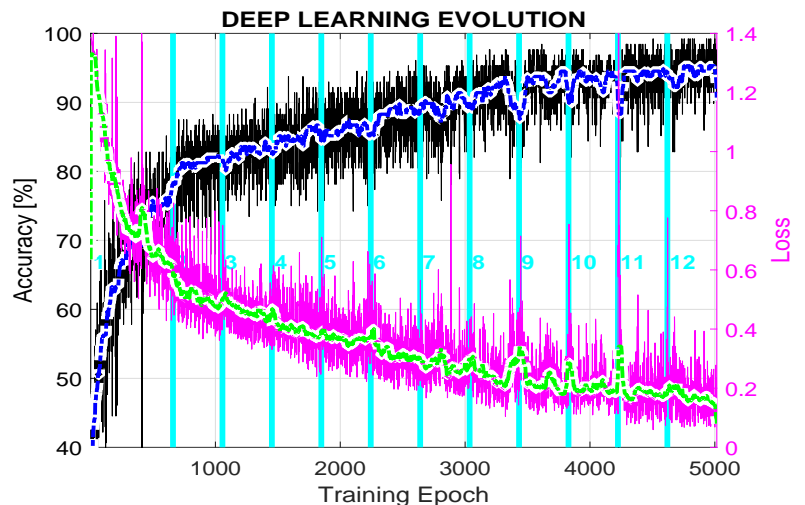

Fig. 5 The evolution of the accuracy and the loss during 12 incremental learning stages of the deep learning system with the final accuracy and loss value $96.9 \%$ and 0.10 , respectively.

$(=0.001)$, learning rate drop period $(=4)$, and gradient threshold $(=2)$ in the incremental mode allowed us to modify the network coefficients during the learning stage. Figure 5 presents the evolution of the accuracy and the loss during 12 incremental learning stages. This process enabled us to improve the behaviour of the DNN with the randomly selected training system initialisations. We achieved the final accuracy and loss value $96.9 \%$ and 0.10 , respectively, after 5000 training epochs. The mean values of accuracies and loss values for last 30 epochs was $97.3 \%$ and 0.09 , respectively. Additional training epochs further increased the accuracy but with the danger of overlearning.

The classification results achieved for the DNN were compared with those evaluated by the SVM method, Bayesian method (Naïve Bayes' Classifier), and the two layer $\mathrm{NN}$ with the sigmoidal and softmax transfer functions. Figure 6 presents the classification into four classes (CA: uphill cycling, CB: downhill cycling, CC: steep uphill cycling, CD: steep downhill cycling) for two features evaluated as the relative power in two frequency

Table 4 Accuracy $A C[\%]$ and the loss value $L V$ for 10 deep learning experiments for different network initialisations presenting the final value (F) after 400 learning epochs and

\begin{tabular}{|c|c|c|c|c|}
\hline \multirow[b]{2}{*}{ Experiment } & \multicolumn{2}{|c|}{ Accuracy [\%] } & \multicolumn{2}{|c|}{ Loss Value } \\
\hline & $F$ & F30 & $F$ & F30 \\
\hline 1 & 73.4 & 74.1 & 0.66 & 0.62 \\
\hline 2 & 73.5 & 70.9 & 0.70 & 0.75 \\
\hline 3 & 67.2 & 69.6 & 0.74 & 0.69 \\
\hline 4 & 78.1 & 77.4 & 0.57 & 0.56 \\
\hline 5 & 74.2 & 74.7 & 0.65 & 0.65 \\
\hline 6 & 81.2 & 75.7 & 0.52 & 0.59 \\
\hline 7 & 82.8 & 75.7 & 0.39 & 0.60 \\
\hline 8 & 77.3 & 76.7 & 0.55 & 0.60 \\
\hline 9 & 75.0 & 74.0 & 0.61 & 0.64 \\
\hline 10 & 75.0 & 77.2 & 0.60 & 0.60 \\
\hline MEAN: & 75.8 & 74.6 & 0.60 & 0.63 \\
\hline STD: & 4.4 & 2.6 & 0.10 & 0.06 \\
\hline
\end{tabular}
its mean (F30) evaluated from 30 last epochs. 

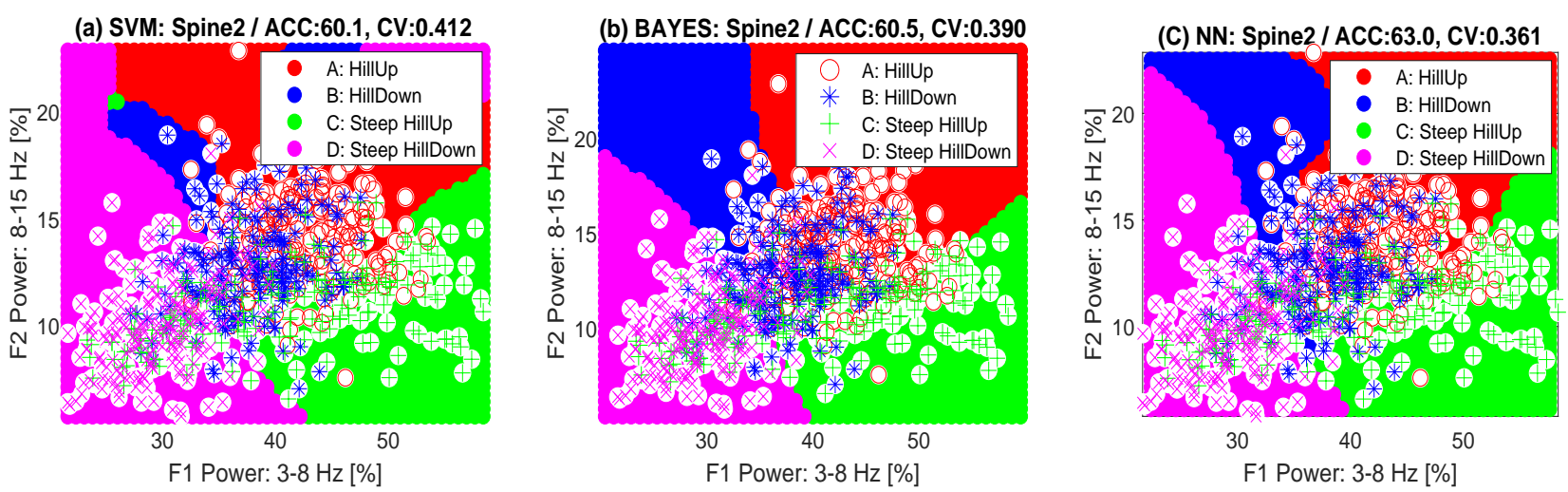

Fig. 6 Classification of accelerometric data for two features evaluated as the relative power in two frequency bands using: (a) the SVM method, (b) Bayesian method, and (c) the two layer NN with accuracy $(A C[\%])$ and cross-validation $(C V)$ errors.

Table 5 Accuracy and cross-validation (CV) errors for classification of accelerometric data into two classes by the SVM, Bayesian method, the two-layer NN and the five-layer DNN.

\begin{tabular}{lcc}
\hline Method & Accuracy [\%] & CV Error \\
\hline SVM Method & 60.1 & 0.412 \\
Bayes' Method & 60.5 & 0.390 \\
2-layers NN & 63.0 & 0.361 \\
\hline 5-layers deep NN & 96.6 & 0.275 \\
\hline
\end{tabular}

bands using selected classification methods with accuracy $(A C)$ and the $k$-fold cross-validation $(C V)$ errors (for $k=10$ ) with visualisation of class boundaries.

Table 5 presents a comparison of the accuracy and cross validation errors for classification of $\mathrm{NN}$ and the five-layer DNN after 5000 learning epochs. Results of classification for the SVM, Bayesian method, and the two-layer NN are very close, with an accuracy between $60.1 \%$ and $62.9 \%$ and the mean value of the crossvalidation of 0.388 . Much higher accuracy of $96.6 \%$ was achieved for the DNN and its cross-validation decreased by $29.1 \%$ to 0.275 .

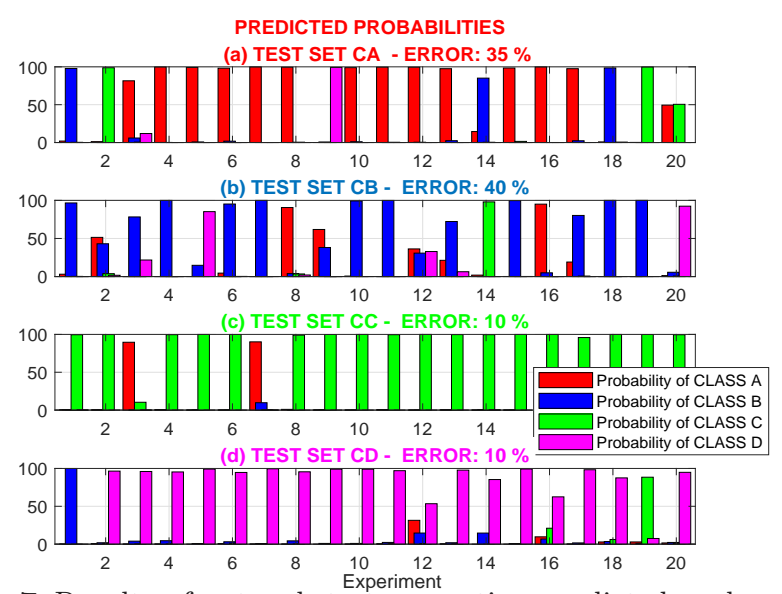

Fig. 7 Results of network test presenting predicted probabilities of classes $\mathrm{CA}, \mathrm{CB}, \mathrm{CC}$, and $\mathrm{CD}$ (corresponding with those in Fig. 6) for the input test set of signal segments that belong to (a) class CA (uphill cycling), (b) class CB (downhill cycling), (c) class CC (steep uphill cycling), and (d) class CD (steep downhill cycling) with associated classification errors.
Figure 7 presents the test of the optimised DL system for input values selected from the testing set recorded for uphill (class CA), downhill (class CB), steep uphill (class CC), and steep downhill (class CD) cycling with the predicted probabilities of of these classes. The mean prediction error is $35,40,10$, and $10 \%$ for individual classes. For each segment four probabilities of the belongings to separate classes are presented with their highest value pointing to the predicted class. The total number of 19 segments out of 80 randomly selected experiments were not classified correctly. In case of the steep slope only 4 segments out of 40 is misclassified.

More detail results for the training and testing sets are presented in confusion matrices presented in Table 6 for the two layer neural network classification into four classes. Similar results evaluated for the deep learning system are presented in Table 7 . The accuracy of the

Table 6 Confusion matrix of the classification by the two layer neural network model for the training and testing sets with true positive values on the matrix diagonal (in the bold).

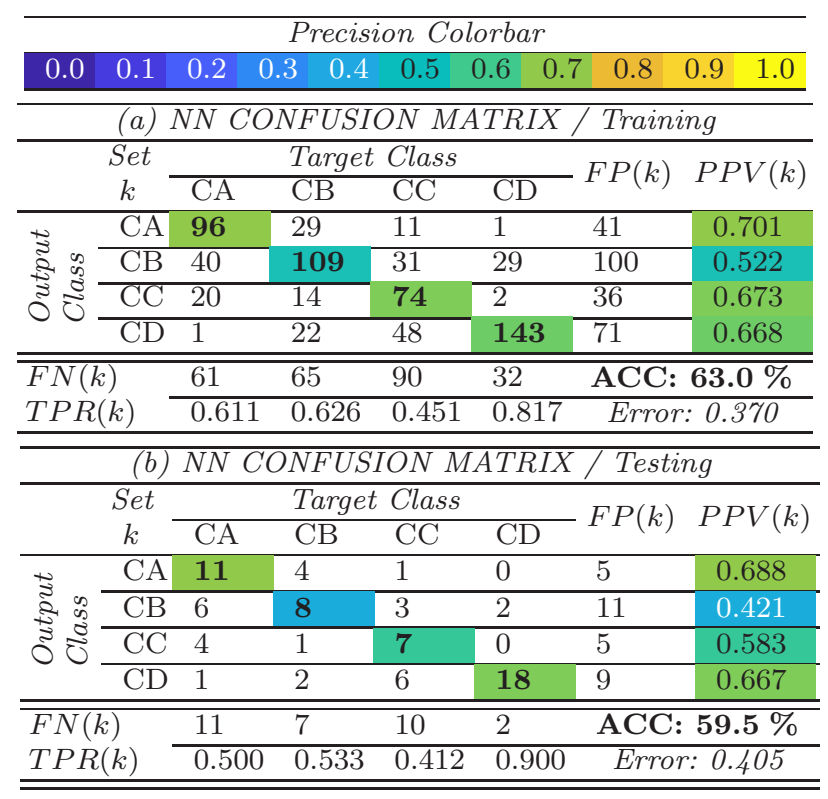


Table 7 Confusion matrix of the classification by the DL neural network model for the training and testing sets with true positive values on the matrix diagonal (in the bold).

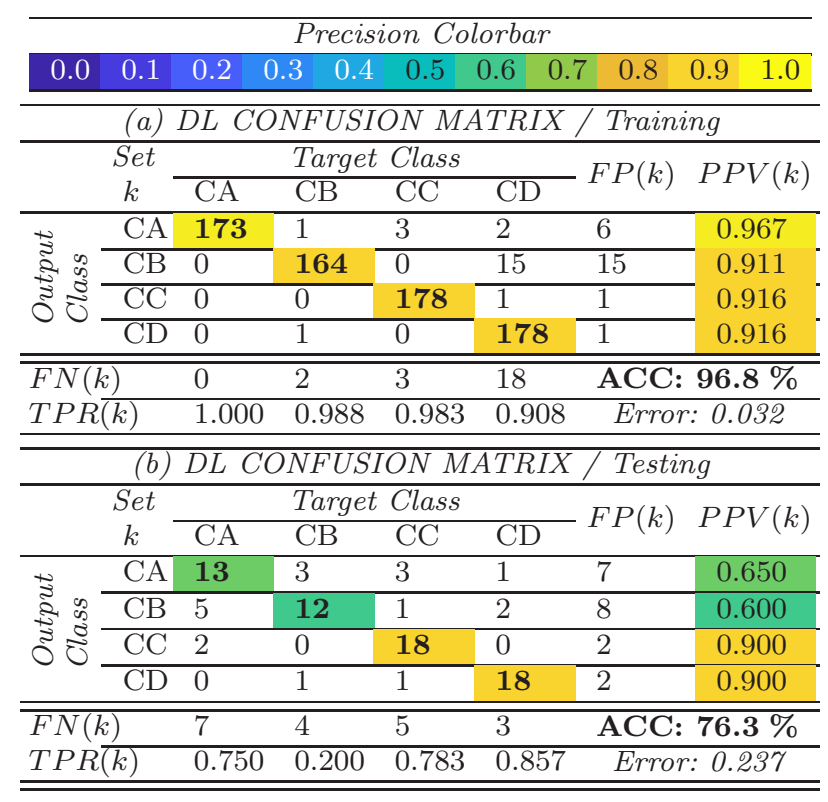

DL system has increased from 63.0 to $96.8 \%$ for the training set. The error value of the testing set decreased from 0.405 to 0.237 . The precision $P P V(k)$ of classes $\mathrm{CC}$ and CD (standing for the steep hill cycling) is much higher than that for classes $\mathrm{CA}$ and $\mathrm{CB}$ allowing better separation of motion activities as assumed.

\section{Discussion}

Our results show that the incremental DNN learning strategy allows continuous monitoring of model accuracy dependent on the level of signal quality, data preprocessing, and selection of model coefficients. Its advantage is moreover in no requirement for selection of signal features. The classification accuracy was $96.6 \%$ and $72.5 \%$ for training and testing sets. Our results show that incremental learning allows continuous monitoring of model accuracy dependent on the level of signal quality, data preprocessing, and selection of model coefficients.

The proposed DNN system used the sigmoidal transfer function in its second layer. Possible improvements can be achieved by different network structures including the use of scaled polynomial constant unit (SPOCU) activation function [30] for instance. Its use increased the training accuracy to $99.3 \%$ (the loss: 0.03).

Classification of motion patterns and monitoring of physical activities by accelerometers is affected by three main factors: (i) optimal body positioning of accelerometric sensors $[13,34,38]$, (ii) the load of the physical exercise presented in Figs. 3, 7, and (iii) computational tools and the selection of the appropriate mathemati- cal methodology. Present results show that higher load during accelerometric data acquisition can contribute to better separation abilities of motion patterns.

The deep learning system can utilize the complete signal segment in the time or transform domain as a pattern vector and thus the process of selection of signal features can be eliminated. Data preprocessing for signal segments classification is simplified as no features are selected but the classification model is much more complex comparing to standard classification methods. In contrary to the deep learning, the standard approach to classification based on a specification of features enables the visualisation of signal segments properties, both in the time and frequency domains. The combination of both approaches to data processing can lead to a deeper understanding of the physical behaviour of the studied system.

The general background of the current research suggests the use of motion patterns classification in many different areas using similar mathematical tools. Featurebased methods can benefit from the visual assessment of their distribution. On the other hand, deep learning methods can be used for the direct analysis of observed sequences either in the time or frequency domains without any initial selection of features but with specific demands for much more complex computational environment. In both cases, new machine learning strategies and their implementation for fitness assessment, for real biomedical data processing, and for monitoring of physiological functions will also be studied.

The video supplement of the present paper includes the animation of several cycling routes related to the (i) accelerometric data acquisition in the time domain for uphill (CA) and downhill (CB) cycling, (ii) their transform into the spectral domain enabling either the direct implementation of the deep learning methodology or evaluation of selected signal characteristics, and (iii) feature-based classification to distinguish two classes of motion activities.

\section{Conclusion}

This paper has presented the use of selected methods for the classification of accelerometric cycling data. Classification into categories of different loads was performed by the DNN with results compared by those of the SVM, Bayesian method and the two layer NN. The advantage of the deep learning is in the fact that no specific features were estimated, the complete data segments were transformed into the frequency domain, and then used as inputs into the proposed model. Limitations of this approach are in the lower possibilities of geometric interpretation of the whole process and needs for the extensive computational environment. 
It is expected that further research will be devoted to the study of specific advantages of deep learning systems for signal and image processing in biomedicine and neurology. The methodological research will include the study of incremental learning strategy allowing to change the system structure and its coefficients during the learning process.

Acknowledgements This research was supported by grant projects of the Ministry of Health, CR (FN HK 00179906) and the Charles University in Prague, CR (PROGRES Q40), by the project PERSONMED - Centre for the Development of Personalized Medicine in Age-Related Diseases, Reg. No. CZ.02.1.01-0.0-0.0-17_048-0007441, co-financed by the ERDF, and grant INTER-ACTION LTAIN19007.

Author Contributions A.P. was responsible for the mathematical and algorithmic tools, H.C. recorded all data and was responsible for their evaluation, O.V. interpreted results from the neurological point of view, D.Y. contributed to data preprocessing, and S.S. contributed to evaluation of results. All authors have read and approved the final manuscript.

Ethical Approval The project was approved by the Local Ethics Committee and all procedures involving human participants were in accordance with the ethical standards of the institutional research committee and with the 1964 Helsinki declaration and its later amendments.

Conflict of interest The authors declare that there is no conflict of interests regarding the publication of this article.

\section{References}

1. Aquino, G., Rubio, J.d.J., Pacheco, J., Gutierrez, G., Ochoa, G., Balcazar, R., Cruz, D., Garcia, E., Novoa, J., Zacarias, A.: Novel Nonlinear Hypothesis for the Delta Parallel Robot Modeling. IEEE Access 8, 46,324-46,334 (2020)

2. Arvidsson, D., Fridolfsson, J., Borjesson, M.: Measurement of physical activity in clinical practice using accelerometers. J. Intern. Med. 286(2), 137-153 (2019)

3. Banan, A., Nasiri, A., Taheri-Garavand, A.: Deep learning-based appearance features extraction for automated carp species identification. Elsevier: Aquacultural Engineering 89, 102,053 (2020)

4. Baron, I., De, J., Rubio, J.d.J., Martinez, D., Vargas, T., Mujica-Vargas, D., Meda Campana, J., Pacheco, J., Gutierrez, G., Zacarias, A.: Genetic Algorithm with Radial Basis Mapping Network for the Electricity Consumption Modeling. Applied Sciences 10, 4239:1-4239:19 (2020)

5. Baumann, T., Hao, T., He, Y., Shoda, R.: Perception Neuron Unity Handbook. Noitom Technology Co., Ltd, 027 edn. (2017)

6. Cao, C., Liu, F., Tan, H., Song, D., Shu, W., Li, W., Zhou, Y., Bo, X., Xie, Z.: Deep Learning and Its Applications in Biomedicine. Genomics, Proteomics \& Bioinformatics 16(1), 17-32 (2018)

7. Carlos, J., Caballero, F., Martinez, F., Hervas, C., Gutierrez, P.: Sensitivity Versus Accuracy in Multiclass Problems Using Memetic Pareto Evolutionary Neural Network. IEEE Transactions on Neural Networks 21(5), 750-770 (2010)

8. Charalambous C. Bharath, A.: A data augmentation methodology for training machine/deep learning gait recognition algorithms. In: Proc. British Machine Vision Conf. (BMVC), pp. 110:1-12. BMVA Press (2016)
9. Charvátová, H., Procházka, A., Vaseghi, S., Vyšata, O., Vališ, M.: GPS-based Analysis of Physical Activities Using Positioning and Heart Rate Cycling Data. SPRINGER: Signal Image Video Process. 11(6), 251-258 (2017)

10. Charvátová, H., Procházka, A., Vyšata, O.: Motion Assessment for Accelerometric and Heart Rate Cycling Data Analysis. MDPI: Sensors 20, 1523:1-1523:13 (2020)

11. Chiang, H., Chen, M., Huang, Y.: Wavelet-Based EEG Processing for Epilepsy Detection Using Fuzzy Entropy and Associative Petri Net. IEEE Access 7, 103,255103,262 (2019)

12. Craik, A., He, Y., Contreras-Vidal, J.: Deep learning for electroencephalogram (EEG) classification tasks: a review. J. Neural Eng. 16, 031,001:1-031,001:28 (2019)

13. Dostál, O., Procházka, A., Vyšata, O., Ťupa, O., Cejnar, P., Vališ, M.: Recognition of Motion Patterns Using Accelerometers for Ataxic Gait Assessment. Neural Computing and Applications 32(x), xxxx-xxxx (2020)

14. Everson, R., Fieldsend, J.: Multi-class ROC analysis from a multi-objective optimisation perspective. Pattern Recognition Letters 27(8), 918-927 (2006)

15. Fan, Y., Xu, K., Wu, H., Zheng, Y., Tao, B.: Spatiotemporal Modeling for Nonlinear Distributed Thermal Processes Based on KL Decomposition, MLP and LSTM Network. IEEE Access 8, 25,111-25,121 (2020)

16. Fawaz, H., Forestier, G., Weber, J., Idoumghar, L., Muller, P.: Deep learning for time series classification: a review. Data Mining and Knowledge Discovery 33, 33:91-963 (2019)

17. Fourcade, A., Khonsari, R.: Deep learning in medical image analysis: A third eye for doctors. Journal of Stomatology, Oral and Maxillofacial Surgery 120(4), 279-288 (2019)

18. Golestani, N., Moghaddam, M.: Human activity recognition using magnetic induction-based motion signals and deep recurrent neural networks. Nature Communications 11, 1551:1-1551:11 (2020)

19. Goodfellow, I., Bengio, Y., Courville, A.: Deep Learning. MIT Press (2016)

20. Gu, Y., Shao, Z., L., Q., Lu, W., Li, L.: A Deep Learning Framework for Cycling Maneuvers Classification. IEEE Access 7, 28,799-28,809 (2019)

21. Guzman-Acevedo, G., Vazquez-Becerra, G., MillanAlmaraz, J., Rodriguez-Lozoya, H., Reyes-Salazar, A., Gaxiola-Camacho, J., Martinez-Felix, C.: GPS, Accelerometer, and Smartphone Fused Smart Sensor for SHM on Real-Scale Bridges. Hindawi: Advances in Civil Engineering 19, 6429,430:1-6429,430:15 (2019)

22. Hassan, M., Uddin, M., Mohamed, A., Almogren, A.: A robust human activity recognition system using smartphone sensors and deep learning. Future Gener. Comp. Syst. 81, 307-313 (2018)

23. He, K., Zhang, X., Ren, S., Sun, J.: Deep residual learning for image recognition. In: Proceedings of the 2016 IEEE Conference on Computer Vision and Pattern Recognition, pp. 770-778. IEEE (2016)

24. Hernendez, G., Zamora, E., Sossa, H., Tellez, G., Furlan, F.: Hybrid neural networks for big data classification. Neurocomputing 390, 327-340 (2020)

25. Huang, Z., Chau, K.: A new image thresholding method based on Gaussian mixture model. Elsevier: Applied Mathematics and Computation 205(2), 899-907 (2008)

26. Jarchi, D., Pope, J., Lee, T., Tamjidi, L., Mirzaei, A., Sanei, S.: A review on accelerometry-based gait analysis and emerging clinical applications. IEEE reviews in biomedical engineering 11, 177-194 (2018) 
27. Jerhotová, E., Švihlík, J., Procházka, A.: Biomedical Image Volumes Denoising via the Wavelet Transform, pp. 435-458. INTECH (2011)

28. Jia, W., Ling, B., Chau, K., Heutte, L.: Palmprint identification using restricted fusion. Elsevier: Applied Mathematics and Computation 205(2), 927-934 (2008)

29. Ker, J., Wang, L., Rao, J., Lim, T.: Deep Learning Applications in Medical Image Analysis. IEEE Access 6, 9375-938 (2018)

30. Kiselak, J., Lu, Y., Švihra, J., Szepe, P., Stehlík, M.: "SPOCU": scaled polynomial constant unit activation function. Neural Computing and Applications xxx, xxx$\mathrm{xxx}(2020)$

31. Konsolakis, K.: Physical Activity Recognition Using Wearable Accelerometers in Controlled and Free-Living Environments. Master thesis, TU Delft (2018)

32. Landgrebe, T., Duin, R.: Approximating the multiclass ROS by pairwise analysis. Pattern Recognition Letters 28, 1747-1758 (2007)

33. Maninni, A., Intille, S., Rosenberger, M., Sabatini, A., Haskell, W.: Activity Recognition Using a Single Accelerometer Placed at the Wrist or Ankle. Medicine and science in sports and exercise 45(11), 2193-2203 (2013)

34. Mannini, A., Intille, S.: Classifier Personalization for Activity Recognition Using Wrist Accelerometers. IEEE Journal of Biomedical and Health Informatics 23(4), 1585-1594 (2019)

35. Meda-Campana, A.: On the Estimation and Control of Nonlinear Systems With Parametric Uncertainties and Noisy Outputs. IEEE Access 6, 31,968-31,973 (2018)

36. Mehrang, S., Pietila, J., Tolonen, J., Helander, E., Jimison, H., Pavel, M., Korhonen, I.: Human Activity Recognition Using A Single Optical Heart Rate Monitoring Wristband Equipped with Triaxial Accelerometer. In: H. Eskola, O. Väisänen, J. Viik, J. Hyttinen (eds.) EMBEC \& NBC 2017, pp. 587-590. Springer Singapore, Singapore (2018)

37. Mirza, B., Wang, W., Wang, J., Choi, H., Chung, N., Ping, P.: Machine Learning and Integrative Analysis of Biomedical Big Data. MDPI: Genes 10, 87:1-87:29 (2019)

38. Montoye, A., Pivarnik, J., Mudd, L., Biswas, S., Pfeiffer, K.: Comparison of activity type classification accuracy from accelerometers worn on the hip, wrists, and thigh in young, apparently healthy adults. Meas. Phys. Educ. Exerc. Sci. 20(3), 173-183 (2016). DOI 10.1080/1091367X.2016.1192038

39. Murad, A., Pyun, J.: Deep Recurrent Neural Networks for Human Activity Recognition. Sensors 17, 2556:12556:17 (17)

40. Neverova, N., Wolf, C., Lacey, G., Fridman, L., Chandra, D., Barbello, B., Taylor, G.: Learning Human Identity From Motion Patterns. IEEE Access 4, 1810-1820 (2016)

41. Nielsen, M.: Neural Networks and Deep Learning. Determination Press (2015)

42. Nishizaki, H. and Makino, K.: Signal Classification Using Deep Learning. In: Proceedings of the 2019 IEEE International Conference on Sensors and Nanotechnology, pp. 1-4. IEEE, Penang, Malaysia (2019)

43. Ordonez, F., Roggen, D.: Deep Convolutional and LSTM Recurrent Neural Networks for Multimodal Wearable Activity Recognition. MDPI: Sensors 16, 115:1-115:25 (2016)

44. Procházka, A., Charvátová, H., Vaseghi, S., Vyšata, O.: Machine Learning in Rehabilitation Assesment for Thermal and Heart Rate Data Processing. IEEE Trans. Neural Syst. Rehabil. Eng. 26(6), 1209-12,141 (2018)
45. Procházka, A., Kuchyňka, J., Vyšata, O., Cejnar, P., Vališ, M., Mař́k, V.: Multi-Class Sleep Stage Analysis and Adaptive Pattern Recognition. MDPI: Applied Sciences 8(5), 697:1-697:14 (2018)

46. Procházka, A., Vaseghi, S., Charvátová, H., Ťupa, O., Vyšata, O.: Cycling Segments Multimodal Analysis and Classification Using Neural Networks. MDPI: Appl. Sci. 7, 581:1-581:11 (2017)

47. Procházka, A., Vyšata, O., Charvátová, H., Vališ, M.: Motion Symmetry Evaluation Using Accelerometers and Energy Distribution. MDPI: Symmetry 11, 2929:12929:13 (2019)

48. Procházka, A., Vyšata, O., Vališ, M., Ťupa, O., Schatz, M., Mařík, V.: Bayesian classification and analysis of gait disorders using image and depth sensors of Microsoft Kinect. Digit. Signal Prog. 47(12), 169-177 (2015)

49. Procházka, A., Vyšata, O., Vališ, M., Ťupa, O., Schatz, M., Mařík, V.: Use of Image and Depth Sensors of the Microsoft Kinect for the Detection of Gait Disorders. SPRINGER: Neural Computing and Applications 26, 1621-1629 (2015)

50. Radu, V., Tong, C., Bhattacharya, S., Lane, N., Mascolo, C., Marina, M., Kafsar, F.: Multimodal Deep Learning for Activity and Context Recognition. In: Proceedings of the ACM on Interactive, Mobile, Wearable and Ubiquitous Technologies, vol. 1, pp. 157:1-157:27 (2017)

51. Raza, A., Mehmood, A., Ullah, S., Ahmad, M., Choi, G., On, B.: Heartbeat Sound Signal Classification Using Deep Learning. MDPI Sensors 19, 4819:1-4819:15 (2019)

52. Rosenberger, M., Haskell, W., Albinali, F., Mota, S., Nawyn, J., Intille, S.: Estimating activity and sedentary behavior from an accelerometer on the hip or wrist. Med. Sci. Sports Exerc. 45(5), 964-975 (2013). DOI 10.1249/MSS.0b013e31827f0d9c

53. Rubio, J.d.J., Rubio, J., Pacheco, J., Gutierrez, G., Ochoa, G., Balcazar, R., Cruz, D., Garcia, E., Novoa, J., Zacarias, A.: SOFMLS: Online Self-Organizing Fuzzy Modified Least-Squares Network. IEEE Transactions on Fuzzy Systems 17(6), 1296-1309 (2009)

54. Sanei, S., Jarchi, D., Constantinides, A.: Body Sensor Networking, Design and Algorithms. Wiley \& Sons (2020)

55. Sangaiah, A.: Deep Learning and Parallel Computing Environment for Bioengineering Systems. Elsevier Science (2019)

56. Shamshirband, S., Rabczuk, T., Chau, T.: A Survey of Deep Learning Techniques: Application in Wind and Solar Energy Resources. IEEE Access 7, 164,650-164,650 (2019)

57. Twomey, N., Diethe, T., Fafoutis, X., Elsts, A., McConville, R., Flach, P., Craddock, I.: A Comprehensive Study of Activity Recognition Using Accelerometers. MDPI: Informatics 5, 5:1-5:37 (2018)

58. Wang, J., Chen, Y., Hao, S., Peng, X., Hu, L.: Deep learning for sensor-based activity recognition: a survey. Pattern Recogn. Lett. 119, 3-11 (2019)

59. Wang, K., Zhou, W.: Pedestrian and cyclist detection based on deep neural network fast R-CNN. SAGE: International Journal of Advanced Robotic Systems 16(2), 1-10 (2019)

60. Yang, J., Xie, Y., Liu, L., Xia, B., Cao, Z., Guo, C.: Automated Dental Image Analysis by Deep Learning on Small Dataset. In: Proceedings of the 42nd IEEE International Conference on Computer Software \& Applications, pp. 492-497. IEEE, Tokyo, Japan (2018)

61. Zhang, J., Chau, K.: Multilayer Ensemble Pruning via Novel Multi-sub-swarm Particle Swarm Optimization. J. Univ. Comp. Sci. 15(4), 840-858 (2009) 